\title{
LA ACADEMIA VALENCIANA (1701-1705) Y LA PERPETUACIÓN DEL BARROCO
}

\author{
Pasqual MAS I USÓ
}

Apenas entrado el siglo XVIII se formó en Valencia la última academia del reinado de los Austrias, pues, tras la muerte de Carlos II, esta ciudad, como el resto de España, va a entrar en un nuevo período que le supondrá centrarse en problemas políticos y bélicos y, consecuentemente, ello llevará a una nueva despreocupación por la cultura. Pero antes del cambio de dinastía real hubo unos años en los que se perpetuó el período Barroco dentro del siglo XVIII, ya casi ahogado en su propio retorcimiento, en todas sus parcela artísticas, y las academias literarias no fueron una excepción'.

Las academias valencianas en el siglo XVII se formalizan bajo dos posibilidades estructurales: como ordinarias o de ocasión, y la Academia de Valencia presenta ambos tipos de formación.

Las academias ordinarias presentan reuniones semanales en las que se tratan los temas propuestos en la sesión anterior por el presidente o por el secretario. Dentro de estas academias existe jerarquización entre sus componentes -desde el presidente al último en ser admitido por el portero-, y de ahí que haya también encargados de preparar los temas que se han de discutir. Asimismo, al estudiar la tendencia de los temas se observa el seguimiento de presupuestos

- Para el estudio de las academias valencianas del siglo XVII ver Pasqual MAS, Justas, Academias y Convocatorias literarias en la Falencia Barroca (1591-1705). Teoría y Práctica de una convención. Tesis Doctoral, Universitat de València, 1991 y "Academias valencianas durante el Barroco" VVAA, De las Academias a la Enciclopedia. El discurso del saber en la modernidad, València, 1992; pág. 153-205. 
poshumanistas enfocados al logro de algúm tipo de aprendizaje al tiempo que se procura diversión y regocijo; es por esto por lo que el lema latino instruire aut delectare se repite en las rescatadas instituciones de algunas academias valencianas.

La primera en adoptar una actitud poshumanista fue la Academia de los Nocturnos (1591-1594) que, a su vez, sirvió de difusora hacia otras manifestaciones académicas como los Montañeses del Parnaso (1615-1616). Y también son casos sintomáticos del auge académico en esta época las academias ficticias que aparecen en obras literarias y en las que la ubicación de su trama histórica es la capital valenciana, en concreto la novela El sutil cordobés Pedro de Urdemales de Alonso Jerónimo de Salas Barbadillo y Huerta de Valencia de Alonso Castillo Solórzano. Todas estas academias de carácter ordinario presentan algunas características comunes:

- reuniones regulares,

- presencia de instituciones que marcan el camino a seguir por los acadénicos,

- jerarquización de los cargos,

- y seguimiento de máximas marales, como umesclar lo dulçe con lo provechoso", que ya apuntaban por primera vez los Nocturnos.

Y las diferencias generales entre ellas afectan a si la academia se desarrolla en la primera o segunda mitad del siglo XVII. Desde finales del XVI hasta las dos primeras decenas del XVII los académicos tienen seudónimos y los cargos con poder dentro de la academia son desempeñados por nobles o personas del alto clero. En cambio, en la segunda mitad del XVII se llega a encontrar como presidente o secretario a José Ortí y Moles, secretario del ayuntamiento de Valencia. Y, aunque la presencia de nobles se mantiene muy elevada, comienzan a entrar en las academias profesores de Universidad, doctores, pintores, bailarines, cantantes e incluso estudiantes que iban a seguir aprendiendo después de sus clases en la Universidtad; institución cada vez más unida a las academias, pues no en vano ambas llegaron a gozar del patronato del ayuntamierto de la ciudad. Támbién se perfila como otro rasgo importantísimo en el desarrollo de las academias valencianas de la segunda ruitud del siglo XVII que dentro de estas asociaciones, con reuniones regulares, se van a celebrar actos literarios con carácter extraordinario que conforman, en realidad, las academias de ocasión. De todas mancras, la característica principal que diferencia a las academias de las dos mitades del XVII es que en las últimas se tiende haciá la especializarión tenática. Si en los Noctumos cualquier tema erá válido para enseñar y divertir (prosa: el caballo, un verso del Ars Amandi de Ovidio, la música, ete; poesía: predominantemente en tormo al amor) ahora se centrarán los temas en sólo unas 
cuantas disciplinas humanísticas y científicas. Además, esta tendencia hacia la especialización afecta al tipo de discurso empleado, ya que en las academias de la segunda parte del seiscientos la prosa se emplea para asuntos seudocientíficos y la poesía queda prácticamente relegada a las celebraciones festivas en las academias de ocasión.

En lo tocante a las academias de ocasión, cabe señalar que éstas se realizan como una función festiva con motivo de ensalzar el honor de algún virrey, de celebrar los años del Carlos II, o la entrada en los territorios de España de Felipe V de Castilla y IV de Aragón.

Durante la primera mitad del siglo XVII tan sólo se tiene noticia de la Academia de la sapiencia de 1606 y de otrá academia celebrada en 1639. Pero cuando comienza el verdadero auge de las academias de ocasión es en la segunda mitad del seiscientos con la llegada de Francisco de la Torre y Sevil y la formación de la Academia de los Soles en 1658 y 1659 . Esta academia presenta estructura parecida a los certámenes literarios: introducción; a veces, cedulillas; poemas, que sólo en Académico Pensil/1669 son en prosa; y, no siempre, vejamen. Pero lo que nunca hay es sentencia. Es decir, frente a los certámenes literarios, ahora no hay concurso; no hay, por lo tanto, premios.

Las academias de ocasión en el último cuarto de siglo, organizadas mayoritariamente dentro de las academias ordinarias, van a potenciar cada vez más el elemento musical, hasta alcanzar grandes cotas de espectacularidad en las tres manifestaciones de la Academia de Valencia en 1703, 1704 y 1705 , donde la academia se lleva a cabo como una representación para-teatral en la que los académicos cantan sus poemas y relaciones de memoria, exceptuando los asuntos en prosa que 110 se debieron cantar. Además, también como característica que delata la tendencia teatralizadora de estas academias de ocasión azarzueladas, se decoraba la sala con gran boato con el fin de provocar asombro y lograr la espectacularidad entre los asistentes, académicos y público, efecto que caracteriza en las artes los últimos años del Barroco.

A la hora de ubicar temporalmente la Academia Valenciana de principios del siglo XVIIJ no se puede pasar xuás allá de la mera especulación, pues si bien existen fechas concretas de las representaciones extraordinarias que se llevaron a cabo en su seno, también es cierto que la academia, desde un tiempo indeterminado que podría alargarse más o menos un año, venía celebrando sus reujnones regulares los fines de senuana. Así pues, de lo único que se tiene certeza es de la realización dentro de ésta de varias fiestas académicas con motivo de recordar la coincidencia de que la fiesta del patrón de Valencia, San Vicente Mártir (21 de enero), esté cercaua al cunpleaños del rey (19 de diciembre). 
Esta Academia Valenciana mantiene la tradición que durante el fin del XVII habían desempeñado las academias con preocupaciones científicas, como en el Alcázar de $1681^{2}$, la Matemática de $1687^{3}$, o la denominada Desamparados-san Javier de $1690^{+}$en las que ya se perciben las tendencias a tratar asuntos de astronomía, álgebra, óptica o matemáticas. Pero, en cambio, en sus actos extraordinarios permanece anclada, como se verá, en el fasto y boato del Barroco más extremo y recargado.

El 19 de diciembre de 1701, cumpleaños del rey, se tomó la primera determinación sobre la creación de una nueva academia; pero se reconoce que, la Academia Valenciana llevaba ya algún tiempo de funcionamiento, por lo que es fácil proponer la conexión directa de la academia Desamparados-san Javier/1690 con la Academia Valenciana/1701, y sirviendo de puente entre las dos la Academia a las señoras 1698; con el referente común de celebrar las sesiones ordinarias en casa privadas y de las fiestas académicas en la Diputación.

Ya en años passados, formada ota Acadenia, sacó a luz alguno de sus festivos empleos, que continuaron hasta que, ocurrencias y variedades de tienpos, suspendieron su continuación; y, en breve distancia de tiempo, los cavalleros de poca edad, que cantayan Tiples, y los que se exercitavan en la Danza passaron a edad menos proporcionada para essos empleos; los mayores, unos, ausentes; otros, en más precissas ocupaciones, [y ello] hizo passar a olvido lo que fue suspensiónt.

La vinculación es, planteada así, estrechísima; y lo fue aunque se trate de academias diferentes que se saben continuadoras de una misma tradición, pero entre la Academia Desamparados-san Javier de 1690 y la Academia Valenciana del XVIII existió un reconocido rompimiento, que evidencia filiación de la segunda con respecto de la primera. De esta vinculación se da fe en la conti-

* La mayoría de los datos de esta academia se encuentran en José ORTÍ Y MOLES, Papeles varios. Ptosa Mathemáticos, Políticos y Jocosos a diferentes assumptas. Escritos Por D. Joseph Orti y Moles y Copiados por ur Sobrino suyo D. Joseph Vicente Ortí y Mayor. Biblioteca Serrano Morales (BSM) Ms. 6564. Y también en José ORTÍ Y MOLES, Aive, tierna y narr son fuego, Ed. de Pasqual Mas i Javier Vellón, Kassel, Reichentuerger, 1992 (Vid. Apéndice).

3 Vid. Juan Bautista GORACHÁN, Avisos de Parnaso, su autor el Dr. Valencia, Viudúd de Antonio Bondaza;, 1747. Los Avisos son de 1690; el libro fué redactadó en 1693 y publicarlo en 1747 gracias a la intervención de Gregorio Mayans.

José ORTÍ Y MOLES, Papeles narios.

5 Academia Valenciana de 1703. Sin portada. (Al fin) En Valencia. Por Vicente Cabrena, Impresor y Librero de la Cludad, en Plaça de la Sea. Año 1703. BSM: A-25/92, pág. 11 (En adelante Academia/1703). 
nuación del primer texto impreso que proporcionó la Academia de Valencia, en 1703. a propósito de la celebración de una fiesta académica:

Y haviendo cessado el confusso tropel de estos años passados, pocos de los que allí concurrían se juntaron para hazer algún estudio de las Mathemáticas, unos para aprender sus primeros rudimentos, otros para delantar las pocas noticias ya adquiridas, y otros para perfeccionar las que ya con el estudio tenían más adelantada[s].

Llamó esta Concurrencia de nuy pocos la compañia de los que ya estavan en la antecedente, y, para la suspensión de la tarea de otros días, destinaron el de los sábados para que suavizasen la fatiga del estudio con la diversión de la Poesía, papeles de Política y Mathemática, Música, Danza, Representación y semejantes ingenios y divertidos empleos, sin más formalidad que lo que a cada uno le ofrecía el genio; y para exercitar la promptitud del discurrir se proponían algunos Problemas para que de repente se respondiera a quien allí se elegía al tiempo de la propuesta

Los documentos son importantísimos, y comentan la relación entre la $A c a-$ demia de Valencia y la ordinaria immediatamente anterior, que fue Desamparados-san Javier ${ }^{7}$, señalándose que algunos de la academia de 1690 formaron ésta de 1701.

Por otra parte, se concretan las recomendaciones de José Ortí y Moles en cuanto a procurar que los estudiantes acudan a sus clases y despues a la academia. La solución encontrada es pasar a las reuniones a los sábados (en Desamparados-san Javier eran jueves) para que "suavizasen la fatiga del estudio" en los entretenimientos de la academia, que, en el caso de predominar los matemáticos, no serían tan descansados.

También se aportan en este texto los temas tratados, que son coincidentes con Desamparados-san Javier, pero esta vez no se mandaban asuntos de una sesión a otra, sino que se confían al "genio» de cada uno para su preparación. Además, también se trataban asuntos «de repente», es decir, se planteaba un problema y se requería a alguien para que lo resolviera en el acto.

Dentro de esta Academia Fulenciana se realizaron tres fiestas académicas azarzueladas. Primeramente, aparte de éstas, el 19 de diciembre de 1701, por

". Acadenia/1703, pág. 12.

T Entre Desamparados-san Javier, que comienza sus sesiones en 1690, y la Acadenia Va. lenciana del XVIII se realizaron varias fiestas académicas: Academia a san Nicolcis de Bari (ca, 1695), Arademia cn casa de Martin Vélonga (ca. 1695) y Academire a Las señoras de 1698 . Vid. los diferentes manuscritos en José Ortí y Moles, Papelés nciros. De la última, prácticamente teatralizada, preparo ahora su cdición. 
el cumpleaños del rey, se iba a celebrar una academia extraordinaria, pero por enfermedad y accidentes de algunos académicos se realizó el 22 de enero de 1702 , día de san Vicente mártir y día en que «se cumplía un año de la Entrada de su Magestad en tierras de España». Esta primera celebración fue como las de todos los sábados:

La forma de Exercicio fuc sólo de aquello mesmo que se componen los de todos los Sábados: papeles de Política y Mathenática, assumptos Poéticos, Música, Representación, y porque ni aun en esto faltasse la formalidad de exercico, no dexaron de proponerse problemas, procurando también fuesen del intento ${ }^{\mathrm{a}}$.

Es decir, se trata de un acto de carácter ordinario, pero se vio la necesidad de preparar algo mayor, y fue entonces cuando se propuso al productivo tándem de José Ortí y Moles y Antonio Ladrón de Pallás, puesto a prueba sobradamente en las fiestas académicas de finales del XVII, para organizar la fiesta del año siguiente. El acto reseñado fue el primer intento de academia extraordinaria, pero no se imprimió y ello determinó que pasara prácticamente desapercibida y ni siquiera para los propios académicos tuviera el rango de fiesta académica. Los trabajos de preparación de la próxima fiesta académica fueron en la Navidad de 1702 y el 22 de enero de $1703^{\circ}$ la Diputación albergó este acto extraordinario por todo lo alto. En esta ocasión sí se trata de la primera fiesta académica, aunque fuera la segunda celebración valenciana de la entrada del rey.

En esta celebración se potenció tanto el carácter teatral de la académica fiesta que los poetas no leyeron sus versos, sino que los recitaron «de memoria a forma de representación*. Además, la música y los poemas cantados experimentaron mayor auge que en la Academia a las señoras 1699 y se formaron dos coros compuestos por tres instrumentistas cada uno (arpa, violón y archilaud), cuatro voces (tenor, alto y dos tiples), y además siete académicos que cantan los asuntos e interlocuciones. No todos los que expusieron un asunto lo habian compuesto, y tan sólo prestaron su voz para recitarlo, aunque sí hubo asuntos matemáticos y laudatorios en prosa que fueron pensados y expresados por quienes los expusieron.

Para llevar a cabo esta fastuosa representación, el palacio de la Diputación se decoró a la altura de los invitados que iban a asistir, pues al acto acudió has-

* Acadermia/1703, pág. 11.

- Acgdemia/1703, pág. 12 . 
ta el virrey de Valencia, el marqués de Villagarcía. La decoración del espacio en el que se desarrolló la academia fue del siguiente modo:

Para lo qual estava la frente del Salón adornado de Colgaduras de Terciopelo y en medio, baxo docel, un Retrato de su Magestad; en la mesma frente, hasta distancia competente, el Estrado para las Señoras, inmediato a la parte d[e]recha, sillas para el Señor Virrey y algunos Cavalleros que estuviessen con su Excelencia, y a la parte correspondiente a ésta, la silla donde, como se dirá en la relación del exercicio, estuvo el Presidente de la Academia. La otra frente del Salón estava dispuesta con algunas gradas para que en ellas y algunos taburetes que se pusieron estuviessen los Cavalleros.

A los lados del salón estavan a una parte el Coro de la música que defendía el sentir de que se celebrassen los Años en puesto algo eminente, para que todo el concurso lo lograsse; y, junto a éste, los que en lo representado y assumptos havían de defender el mismo dictamen; a la otra parte correspondiente el otro Coro de la Música y demás que defendían fuesse la Celebración a la Entrada y arribo de su Magestad a España... ${ }^{10}$.

Durante el año de 1703 la Academia de Valencia siguió reuniéndose para tratar sus asuntos ordinarios, y de ello se encuentra la prueba en algunos manuscritos de José Ortí y Moles, como el Papel Mathemático para uno de los ordinarios exercicios de la Academia año 1703, sobre la ruina que amenazó el campanario de san Bartolomé en el discurso de su fábrica ${ }^{11}$ y también en Primer día de Exercicio de la Academia en la Presidencia de D. Juan Ruiz de Corella y Vergadá; y superintendencia de la Mathemáticas de D. Joseph Ortí. Academia en el día 16 de Junio $1703^{12}$. Este último texto da además la noticia del cambio de presidente, cargo desempeñado hasta entonces por José Pascual de la Verónica, y a partir de enero de 1705 por el conde del Castellar, Nicolás Felipe de Castelví Vilanova.

La continuidad de la Academia Valenciana estaba asegurada por las reuniones periódicas en las que los asuntos no eran predominantemente de carácter literario. En este sentido se puede constatar una continuidad estructural con las academias ordinarias, a la manera de los Nocturnos (1591-1594) o del Alcázar (desde 1681), pero también un cambio en cuanto a los temas abordados y, en este sentido conecta con la mencionada Academia Matemática/1687. El siguiente texto es muestra de la reflexión referida:

... aunque es verdad que dissuena del principal intento para que fue fundada la Academia, que es el de la Mathemática... ${ }^{13}$.

\footnotetext{
10 Academia/1703, pág. 14-15.

1 José ORTÍ Y MOLES, Papeles varios, pág. 115-119.

12 José ORTÍ Y MOLES, Papeles varios, pág. 119-126.

13 Academia/1703, pág. 12.
} 
Esta puntualización de la academia de 1703 pone a flote una aparente contradición que, por otra parte, se justifica en tanto que a menudo los académicos, que se reunian al margen de la Universidad, eran vistos como personajes clandestinos y las fiestas acadénicas se convertían en actos de afimación del régimen absolutista y, sobre todo, católico. Esta apariencia de clandestinidad no era excesiva en la Academia Valenciana de principios del XVIII, pues entre sus componentes abundan personajes de lia nobleza. En cambio, en los años 80 del siglo XVII los componentes de la Academia Matemática/1687 o de Desamaparados san Javier 1690 , si tuvieron que mantener a escondidas sus proyectos y discusiones (la circulación de la sangre, el saber de Galileo, los atlas anatómicos copiados en París, etc.). Realmente en los últimos años del XVII surge en Valencia un movimiento renovador, formado por los llamados «novatores» que, al margen de las enseñanzas institucionales y conectados con el resto de Europa, no cesaron de investigar y reflexionar de manera crítica sobre los problemas científicos que se plantearon en la época ${ }^{14}$. Sin duda, el magma dieriochesco de la luz y de la ciencia se encuentra en estas fechas pero, como se puede observar con la existencia de la academia que ahora se analiza, el Barroco se perpetúa hasta entrado el XVIII.

I a segunda fiesta académica debía realizarse e] 22 de enero de 1704 , pero se retrasó al 2 de febrero porque el palacio de la Diputación estaba todavía engalanado con los adornos de la academia anterior. Fste retraso es lamentable por dos cuestiones; la primera porque trastoma el ritmo de lá academia, y la sogunda porque evidencia que la Diputación xo funcionaba demasiado bien en el terreno político, pues resulta evidente que el palacio sólo se utilizaba para fiestas.

Esta segunda fiesta académica ${ }^{15}$ fue un calco de la primera y lo mismo ocu-

it Para el conocimiento de esta época som importantes, entre otros, los siguientes estudios: Vicente PESET, "La Universidad de Vulencia y la renuvación científica españula (1678-1727)", Boletin de la Sociedad Cristellonense de Cultura (1960), XLII, pág. 70-79; Sebastián GARCIA

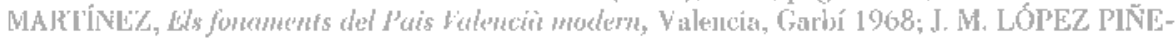
RO, "Los comienzos de la Medicina y las Ciencias Modernas en Lispaña en el último tercio del siglo XVII", Actes del Segundo Congresu de Hestorio de la Medicina, Salamanca, 1965; Ramón CENAL. "Cartesiarisnu) en España. Notos para su historia $(1650-1750)$, Revista de la Lniversidad de Oviedo (1945), pág. 3-97; Autonio MESTRE, El mundo intelectual de Metgons, Valeneia, Ayuntamiento de Oliva, 1978; y Victor NAVARRO BROTONS, Tradició i canvi cientific al Pais Valencià mo-

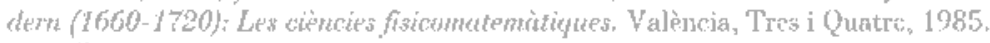

1. Descrija en la Academiat de Valencia en celebración de la gloriosa entrade en los dominios de Espuña, y feliz cumplimiento de años det rer, nuestro señor Don Felipe IV de Aragón y $V$ de Custilla. Efectuada en la cusa de la Diquatación del Reyno de Valencia. El dia 2 de Febrem 1704 . Y

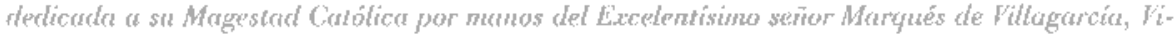
srey y capitán general del Reyno. Por Ficente Cabrera, Impressor y Librero de la Caudad en la plaça 
rrió con la del 22 de enero de $1705^{16}$. En la última hay que resaltar que, según indicación de José Vicente Ortí y Mayor la decoración representaba un Arcadia. Esta idea del sobrino de José Ortí y Moles, por lo que respecta al ámbito valenciano, se había llevado a cabo en la novela El Prado de Valencia de Gaspar Mercader (Valencia, 1600) ${ }^{17}$, y en la realidad también existe un claro precedente, más cercano en el tiempo, en ltalia, ya que en Roma se formó en 1690 la Academia degli Arcadi, cuyos miembros, que llegaron a ser unos 600, tenían por finalidad resucitar el estudio de la poesía en reuniones de enmascarados vestidos de pastores de Arcadia ${ }^{18}$. Y así,

Este generoso deseo dio motivo a la idea que se discurrió de fingirse transformada en Arcadia la Academia, pues siendo la Arcadia Teatro de los cariños, pareció peculiar Idea para expressión de los afectos ${ }^{19}$.

En la Academia de Valencia se intenta justificar en la introducción y los primeros diálogos el porqué de la comparación de la academia con la Arcadia. y se llega al punto de convocar a los académicos como «arcades».

Como se puede apreciar, la Academia de Valencia de 1705 conduce al máximo exponente teatral de las academias; pues, si llegaron al extremo de disfra-

de la Seo. Año 1704. En BSM: A-25-92. (En adelante Academia/1704). Tambićn hay noticias de la academia en el manuscrito de los hermanos Ortí y Moles: "Ln la Academia de Valencia que se celebró en la Casa de la Diputación año de 1704, en cetebración de la gloriosa entrada de los dominios de España y feliz cumplimiento de años del rey $n^{t r}$ st: $V^{n}$ fetipe $V$ (caya t'iesta se imprimió), se le dio por assumpto al autor leyese un papet Mathemático, cuyo assumpto era: Discurrase por el dia y hora del nazimiento del rey $n^{\text {rv }} S^{2}$ la propiedad de aplandir el Turia su gallandia y afabilidad. Pero antes de empezar su papel, le introduvo con los siguientes pareados hablando entre los dos sobrinos suros que eran don Marco Antonio y don Francisco Ortí Figuerola, levantándose el autor apenas se acabó de cantar un Duo, y como si le huviessch llamato, dixo de esta suerte su so. brino". En José ORTÍ Y MOLES, Papeles rarios, pág. 409-417. Ese mismo año también se celebraton otros acontecimientos como el reseñado en el Pupel que en el día seis de agosto $170+$ se leyó en la Academin de la Cudad de Valencia por la feliz noticia del dicho arribo de su Magestad (que Lios guturte) a su corte, triunfante de Partugal. En BSM: A-2!3-92.

16 Festivos obsequios conque acreditó su feliculad la Acalemia de Valencia cetebrando los augustos años y Felize Entrata de el Rey nuestro Señor Don Folipe IV de Aragón y $I$ de Castilla. Executáronse en la Casa de la Diputación de la misma Ciudad y Reyno. En 22 de enero 1705. Y se dedican a su Magestad Cathólica por medio Del Exa Señor Marqués de Villagarcía, Firrery Capitán General de este Regno. En Vatencia en la Imprenta de Vicente Cabrem, en la Placa de la Seo. Año 1705. En BSM: A-25-92. (En adelante Acadenia/1705).

17 Pasqual MAS, "Poetas bajo nombres de pastores en El Prado de Falencia de Gaspar Mercader" Revisla de Literatura LIV, n. ${ }^{\circ} 107,1992$, pág. 283-334.

in Encyclopedia Británica, Cambridge, 1910. T. I, pág. 103.

19. Academia/1705, pág. 10. 
zarse de pastores arcádicos, de ahí al teatro la frontera es bien fina. De hecho, la Academia a las Señoras de 1698 es ya prácticamente una zarzuela, cuyo precedente se encuentra en la Acadenia a la condesa de Peñalba de $1685^{20}$. Así pues, en las tres funciones académico-festivas de la Academica de Valencia es evidente cómo la preocupación por causar asombro se acentúa.

Pero no hay que juzgar sólo a la Academia de Valencia por las tres celebraciones académicas festivas que organizó y que después se imprimieron, ya que esta academia siempre insiste en su dedicación científica y erudita, sobre todo, en relación a las matemáticas; hasta en el decurso de las fiestas académicas se tratan asuntos matemáticos, $\mathrm{y}_{4}$ como se ha visto arriba, no se pierde la oportunidad de recalcar que la academia se formó para aprender y practicar asuntos de esta índole, aunque por el camino se le cruzó el «deber» de realizar fiestas académicas para que el virrey de Valencia pudiera agasajar a Felipe V de Castilla y IV de Aragón, apoyado por la Ciudad y la Diputación ${ }^{21}$ en la Guerra de Sucesión.

Así pues, la estrategia seguida por la Academia de Valencia demuestra que sus componentes gozaban de una inquietud por el estudio sin censuras y que supieron ver que la única posibilidad de seguir adelante con sus proyectos era actuando cara al público como si nada fuera a cambiar jamás. De este modo, los académicos podían continuar sus sesiones y tertulias privadas con la tranquilidad de saberse respaldados por su actuación pública. Es decir, este último Barroco se encuentra doblemente hueco; por una parte el sentido barroco se deja llevar por lo recargado de las formas dejando en el vacío al significado, ya atomizado en la plurisignificación conceptista, ya minimalizado en su tensión cultista, y por otra parte estos académicos que apuntan hacia una manera de pensar nueva comprenden que para que les dejen operar sin interrupciones, han de perpetuar ese modo de ver la realidad desde la abundancia.

La existencia de la Academia de Valencia y, sobre todo el comportamiento de sus componentes, ayuda a comprender que el cambio dinástico no tuvo que ver en la renovación del pensamiento español. El germen ya estaba presen-

20 Academia que se celebró en la ciudad de Balencia en la Atcaydía del Real Palacio, casa de don Luys Itun de Torres y Centellas,... dedicada a... doña Juana Manuela Mingot y Racafull, condesa de Peñatu, dec, siendo secretario Francisco Figuerola, fiscal don Josef Orti. En 5 de Febrero 1685. Valencia, Vicente Cabrera, 1685. En la página 9 se dice respecto al componente para-teatral de esta representación: "¿Qujén eres, triste voz, que te desvelas / en pasar de Academias a Zarçuelas?".

21 Vicent RIVES, Et segle XWH. Alfons el Magnànim, València, 1985; pág. 10 y ss. 
te, simplemente necesitaba planificación, axiomatización: la erudición ya se recogía aunque en silvas, polyanteas y libros del saber, pero no en la Enciclopedia.

El Barroco de estos años es, pues, más que una globalidad de miradas focalizadas en lo exagerado, un recurso que se relame en su decadencia como la única salida posible para aguantar los embates de un período de transición, permeable y ecléctico. Sin duda, es en las últimas academias barrocas, y en gran medida las valencianas, en donde hay que buscar el pulso de lo que más tarde será el pensamiento ilustrado español. 